\title{
Nuevos criterios para el diagnóstico y tratamiento de la trombosis venosa profunda de los miembros inferiores
}

\author{
F. GABRIEL BOTELLA, M. LABIÓS GÓMEZ
}

Servicio de Medicina Interna. Hospital Clínico Universitario. Valencia

\author{
NEW CRITERIA FOR THE DIAGNOSTIC AND TREATMENT OF THE \\ DEEP VEIN THROMBOSIS OF THE LOWER LIMBS
}

\section{RESUMEN}

El diagnóstico de la trombosis venosa profunda (TVP) basado exclusivamente en su sintomatología y en el examen clinico es de poca utilidad debido a su escasa sensibilidad y especificidad. Sin embargo, su adecuada combinación con la presencia de factores de riesgo, y la existencia o no de un diagnóstico alternativo, puede emplearse para clasificar a los pacientes según el modelo de predicción clínica de TVP de Wells en dos categorías: alta o baja probabilidad de padecerla. Su utilización en urgencias junto con la determinación del dímero-D (DD) nos indicará la necesidad o no de realizar un eco-Doppler, actualmente la prueba complementaria de elección. La flebografía se mantiene como "la prueba de oro", aunque se reserva para ciertos casos. Salvo excepciones, las heparinas de bajo peso molecular han desplazado a las no fraccionadas en el tratamiento de la enfermedad, debido a la serie de inconvenientes que presentan: complicaciones hemorrágicas, trombopenia, necesidad de hospitalización y control biológico. Es posible, que en un futuro más o menos próximo nuevos antitrombóticos, como el fondaparinux o el ximelagatrán cambien substancialmente el tratamiento de la TVP. Aunque no están exentos de complicaciones, existen situaciones en las que está indicada la colocación de un filtro en la vena cava inferior. La trombolisis es poco aceptada, debido a que la relación riesgo-beneficio no es satisfactoria. Una excepción, serían las trombosis venosas masivas con riesgo de gangrena (flegmasia cerúlea dolens), en pacientes con bajo riesgo hemorrágico. Finalmente, en los casos que cursan con isquemia venosa, que generalmente aparecen en las oclusiones más proximalesvena iliaca y cava inferior-, una opción eficaz para restablecer la permeabilidad venosa sería la trombectomía venosa.

PALABRAS CLAVE: Trombosis venosa profunda. Modelo de predicción clínica de trombosis venosa profunda. Dímero-D. Eco-Doppler. Heparinas de bajo peso molecular. Trombolisis. Trombectomía venosa

\section{ABSTRACT}

The diagnosis of the deep vein thrombosis (DVT) based exclusively on its symptomatology and on the examination clinic is of few usefulness due to its small sensibility and especificity. However, its adequate combination with the presence of risk factors for the thrombosis, and the existence or not of an alternative diagnosis, it can be employed it to classify to the patients according to the forecast model clinic of DVT of Wells in two categories: high or probability decrease of suffering it. Their utilization in urgencies together with the determination of the $D$ dimer $(D D)$ will indicate us the need or not of accomplishing an echo - Doppler, currently the complementary election test. The phlebography is maintained as "the gold test", though is reservation for certain cases. Unless exceptions, the heparins of under molecular weight have displaced the heparins not fractioned in the treatment of the disease, due to the series of inconvenients that present: complications hemorrhagics, thrombocytopenia, need of hospitalization and of biological control. It is possible, that in a future most or less next new antithrombotics, as the fondaparinux or the ximelagatran change substantially the treatment of the DVT. The indication of the thrombolysis is little accepted, due to the fact that the relationship risk - benefit is not satisfactory. Though they are not exempt of complications, exist clean-looking situations in those which is indicated the placement of a filter in the inferior vein cava. An exception, they would be the thrombosis massive veins with gangrene risk phlegmasia cerulea dolens, in patient with under risk hemorrhagic. Finally, in the cases that developed ischemia venous, that generally appear in the occlusions most proximals - iliac and inferior vein cava -, an effective option to re-establish the venous permeability would be the thrombectomy venous.

KEY WORDS: Deep venous thrombosis. Clinical model for predicting of deep vein thrombosis. D-dimer. Echo-Doppler. Heparins of under molecular weight. Thrombolysis. Thrombectomy venous.

Gabriel Botella F, Labiós Gómez. M. Nuevos criterios para el diagnóstico y tratamiento de la trombosis venosa profunda de los miembros inferiores. An Med Interna (Madrid) 2004; 21: 400-407.

\section{INTRODUCCIÓN}

En todo paciente con sospecha de TVP los signos y síntomas clínicos son fundamentales (1). Sin embargo, son poco sensibles (muchas trombosis dan signos y síntomas muy dis- cretos, o incluso no los dan) y poco específicos (muchas entidades clínicas pueden dar una sintomatología similar a la de la trombosis). A pesar de todo ello, los clínicos disponemos de una información adicional que nos puede ser de gran utilidad ante la sospecha de una TVP. Por una parte los factores de ries-

Trabajo aceptado: 26 de marzo de 2004 
go, esto es, las distintas situaciones clínicas que hacen posible el desarrollo de una trombosis. Por otra, los diagnósticos alternativos a la misma, ya que los síntomas que presenta el paciente pueden ser debidos a otras enfermedades, como la trombosis venosa superficial, el síndrome postrombótico, el quiste de Backer, el linfedema, el hematoma de la pantorrilla, los edemas sistémicos, o una compresión venosa extrínseca. La adecuada combinación de la sintomatología, la exploración física, la presencia de factores de riesgo para la trombosis, y la existencia o no de un diagnóstico alternativo, podemos utilizarla para clasificar a los pacientes, siguiendo el último modelo de predicción clínica de TVP de Wells et al (2) en dos categorías: alta o baja probabilidad de padecerla (Tabla I).

\section{TABLA I}

\section{MODELO DE PREDICCIÓN CLÍNICA DE TVP}

\begin{tabular}{lc}
\hline CARACTERÍSTICAS CLÍNICAS & PUNTOS \\
\hline Cáncer activo (paciente que haya recibido tratamiento & \\
anticanceroso dentro de los 6 meses previos, o que esté & 1 \\
recibiendo tratamiento paliativo ) & \\
Parálisis, paresia o inmovilización reciente con escayola de & 1 \\
los miembros inferiores & \\
Encamamiento reciente > 3 días, o cirugía mayor dentro de las & \\
12 semanas previas a la TVP, ya sea con anestesia regional & 1 \\
o general & \\
Hipersensibilidad localizada a lo largo de la distribución de & 1 \\
las venas del sistema venoso profundo & 1 \\
Edema en todo el miembro afectado & \\
Edema de pantorrilla con fovea, por lo menos 3 cm mayor que & \\
en el miembro asintomático, medido 10 cm por debajo de la & \\
tuberosidad tibial & 1 \\
Edema con fovea limitado al miembro sintomático & 1 \\
Venas superficiales colaterales (no varicosas) & 1 \\
Trombosis venosa profunda previa documentada & 1 \\
Diagnóstico alternativo por lo menos tan probable como la TVP & -2 \\
\hline A
\end{tabular}

Alta probabilidad $>2$ puntos $\quad$ Baja probabilidad $<2$ puntos

*En pacientes con síntomas en ambos miembros, se debe de puntuar el más afectado.

\section{DIAGNÓSTICO DE LA TVP}

El diagnóstico de la TVP se inicia con la sospecha clínica (sintomatología). A continuación la estrategia diagnóstica incluye un modelo predictivo (test de Wells) y la prueba del dímero-D. Sus resultados indicarán la necesidad o no de realizar las exploraciones complementarias pertinentes que confirmen la presunción diagnóstica

\section{EL DÍMERO D (DD) EN EL DIAGNÓSTICO DE LA TVP (3)}

La elevación de la concentración plasmática del DD (productos de la degradación de la fibrina) no es específica de la TVP, sin embargo, se ha demostrado que su normalidad si es de utilidad.

Los métodos de enzimoinmunoanálisis (ELISA) -el método de referencia- y algunos de los recientes métodos de diagnóstico cuantitativo rápido - de utilidad en urgencias- basados en la aglutinación de partículas de látex recubiertas de anticuerpos monoclonales frente al DD, han demostrado su eficacia. $\mathrm{Su}$ elevada sensibilidad (95,5-100\%) y valor predictivo negativo $(>95 \%)$ cuando se emplean reactivos adecuados, permite excluir con un alto grado de fiabilidad la existencia de una TVP.

\section{EL ECO-DOPPLER EN EL DIAGNÓSTICO DE LA TVP(4)}

Actualmente, el eco-Doppler es la prueba complementaria de primera elección después del DD. Además, es rápida, no invasiva y permite exploraciones seriadas. Su valor predictivo positivo puede alcanzar el $97 \%$. La flebografía se mantiene como la "prueba de oro", aunque se reserva para casos dudosos, necesidad de confirmación diagnóstica, evaluación de la trombolisis, inserción de filtros de cava, o para investigación clínica. En las trombosis venosas proximales, su sensibilidad y especificidad es del $96 \%$, en las distales la sensibilidad es del $80 \%$. Las modernas técnicas de imagen, como la tomografía computarizada (TC), la resonancia magnética $(\mathrm{RM})$, y las isotópicas, tienen un futuro prometedor, si bien se encuentran en fase de validación.

La imagen directa del trombo venoso, y de sus defectos vasculares, es posible mediante RM. Este método no invasor, es bien tolerado, reproducible y exacto por debajo de la rodilla, hasta el punto de ser comparable en las imágenes con la flebografía. Conjuntamente se están evaluando, con resultados prometedores, la seguridad y tolerabilidad de diferentes contrastes para realizar RM-venografías. La TC helicoidal ha sido utilizada para detectar TVP en pacientes con sospecha de embolismos pulmonares. La combinación de venografía-TC y escáner pulmonar parece ser un método preciso para el diagnóstico de TVP y embolismos pulmonares.

En resumen, en aquellos pacientes con un modelo clínico predictivo de baja probabilidad más un dímero D (DD) negativo, se puede excluir la TVP, por lo que no es necesario la realización de un eco-Doppler. En todos los demás pacientes con un modelo clínico de alta o baja probabilidad y DD positivo, se debe efectuar un eco-Doppler para confirmar o excluir la TVP (Fig. 1).

\section{TRATAMIENTO DE LA TVP $(5,6)$}

\section{OBJETIVOS}

A corto plazo: prevenir la extensión del trombo, evitar la embolización pulmonar y disminuir el riesgo de recurrencias.

A largo plazo: disminuir las recidivas tardías, evitar el SPT y la aparición de hipertensión pulmonar

TIPOS DE HEPARINAS UTILIZADAS EN EL TRATAMIENTO DE LA TVP

Heparinas de bajo peso molecular (HBPM): Como norma general, se deben de emplear en todos los casos de TVP, durante un período de $5 \pm 2$ días. La administración de anticoagulantes orales (AO) debe iniciarse entre los días 1 y 3 después del inicio de tratamiento con HBPM, simultaneando ambos tratamientos, durante 3-5 días y deteniendo la heparinoterapia cuando se haya alcanzado un INR de 2-3, durante dos días consecutivos. En la tabla II se recogen las dosifica- 


\section{ALGORITMO DE DIAGNÓSTICO DE LA TVP}

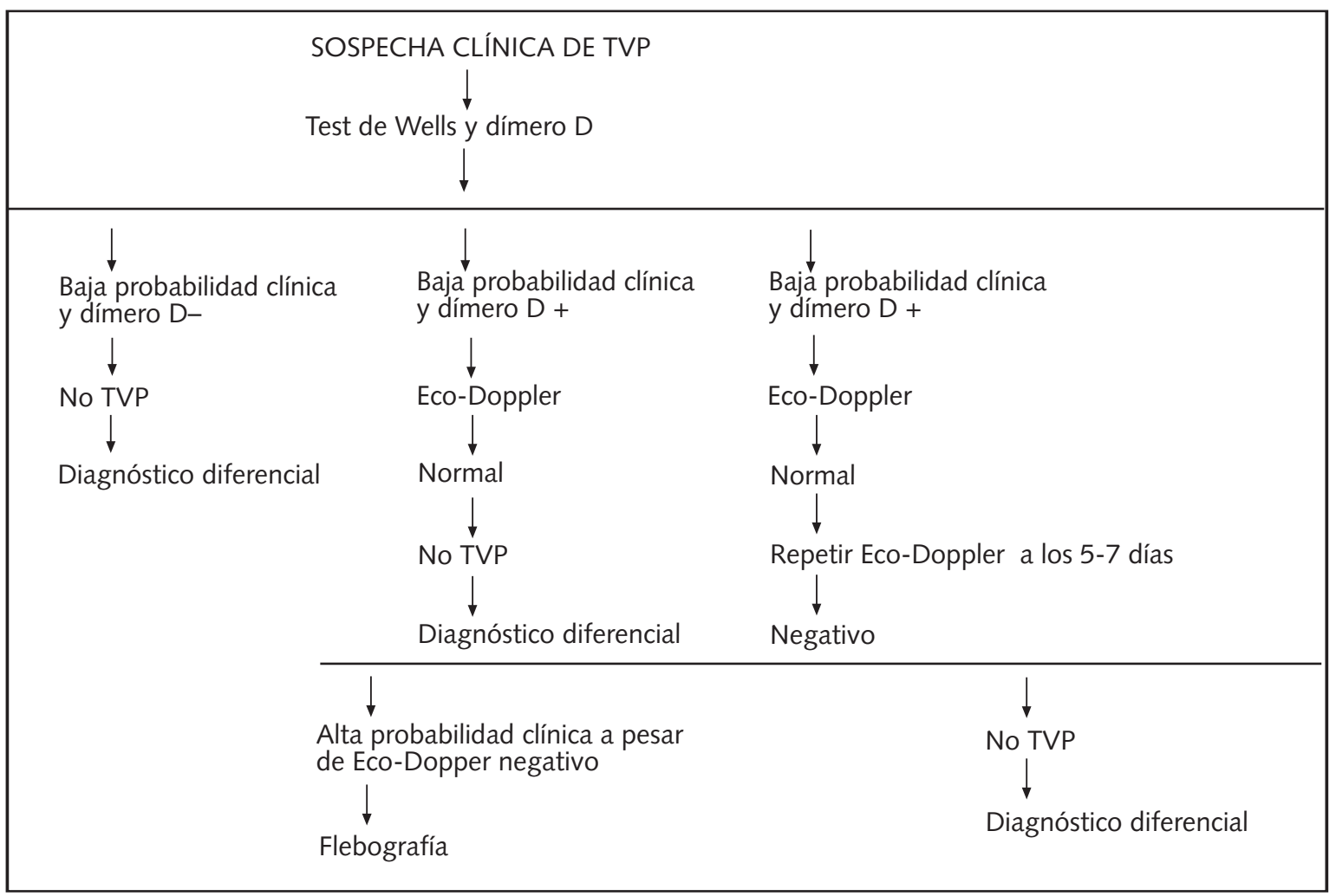

Fig. 1.

\section{TABLA II}

DOSIFICACIÓN DE LAS HBPM EN EL TRATAMIENTO DE LA FASE AGUDA DE LA TVP

\begin{tabular}{|c|c|c|c|c|c|}
\hline Pacientes & HBPM de $2^{a}$ gene & ción & HBPM de $1^{a}$ & ración & \\
\hline con: & Bemiparina & Enoxaparina & Dalteparina & Nadroparina & Tinzaparina \\
\hline$<50 \mathrm{~kg}$. & $\begin{array}{c}5.000 \mathrm{UI} \\
0,2 \mathrm{ml} / 24 \mathrm{~h}\end{array}$ & & $\begin{array}{c}5.000 \mathrm{Ul} \\
(0,2 \mathrm{ml}) / 12 \mathrm{~h} \\
10.000 \mathrm{Ul} \\
(0,4 \mathrm{ml}) / 24 \mathrm{~h}\end{array}$ & $\begin{array}{c}0,4 \mathrm{ml} \\
(3.800 \mathrm{Ul}) / 12 \mathrm{~h}\end{array}$ & \\
\hline $50-70 \mathrm{~kg}$. & $\begin{array}{c}7.500 \mathrm{UI} \\
0,3 \mathrm{ml} / 24 \mathrm{~h}\end{array}$ & $\begin{array}{c}60 \mathrm{mg} \\
(6.000 \mathrm{Ul}) \\
0,6 \mathrm{ml} / 12 \mathrm{~h} \\
\mathrm{~F} 90 \mathrm{mg} \\
(9.000 \mathrm{Ul}) \\
0,6 \mathrm{ml} / 24 \mathrm{~h}\end{array}$ & $\begin{array}{c}12.500 \mathrm{Ul} \\
(0,5 \mathrm{ml}) / 24 \mathrm{~h}\end{array}$ & $\begin{array}{c}0,6 \mathrm{ml} \\
(5.700 \mathrm{UI}) / 12 \mathrm{~h} \\
\mathrm{~F} 0,6 \mathrm{ml} \\
(11.400 \mathrm{Ul}) / 24 \mathrm{~h}\end{array}$ & $\begin{array}{c}0,5 \mathrm{ml} \\
(10.000 \mathrm{UI}) / 24 \mathrm{~h}\end{array}$ \\
\hline$>70 \mathrm{~kg} .\left(^{*}\right)$ & $\begin{array}{c}10.000 \mathrm{Ul} \\
0,4 \mathrm{ml} / 24 \mathrm{~h}\end{array}$ & $\begin{array}{c}80 \mathrm{mg}(8.000 \mathrm{UI}) \\
0,8 \mathrm{ml} / 12 \mathrm{~h} \\
\mathrm{~F} 120 \mathrm{mg}(12.000 \mathrm{UI}) \\
0,8 \mathrm{ml} / 24 \mathrm{~h}\end{array}$ & $\begin{array}{c}7.500 \mathrm{Ul} \\
(0,3 \mathrm{ml}) / 12 \mathrm{~h} \\
15.000 \mathrm{Ul} \\
(0,6 \mathrm{Ul}) / 24 \mathrm{~h} \\
18.000 \mathrm{Ul} \\
(0,72 \mathrm{ml}) / 24 \mathrm{~h}\end{array}$ & 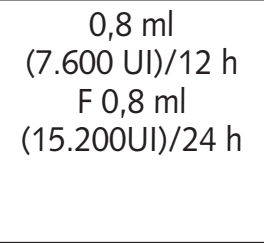 & $\begin{array}{c}0,7 \mathrm{ml} \\
(14.000 \mathrm{Ul}) 24 \mathrm{~h}\end{array}$ \\
\hline $\begin{array}{l}\text { Posología } \\
\text { recomendada }\end{array}$ & $115 \mathrm{UI} / \mathrm{kg} / 24 \mathrm{~h}$ & $\begin{array}{c}1 \mathrm{mg}(100 \mathrm{UI}) / \\
\mathrm{kg} / 12 \mathrm{~h} \\
\mathrm{~F} 1,5 \mathrm{mg} \\
(150 \mathrm{UI}) / \mathrm{kg} / 24 \mathrm{~h}\end{array}$ & $\begin{array}{l}100 \mathrm{UI} / \mathrm{kg} / 12 \mathrm{~h} \\
200 \mathrm{UI} / \mathrm{kg} / 24 \mathrm{~h}\end{array}$ & $\begin{array}{c}85,5 \mathrm{Ul} / \mathrm{kg} / 12 \mathrm{~h} \\
\mathrm{~F} 171 \mathrm{Ul} / \mathrm{kg} / 24 \mathrm{~h}\end{array}$ & $175 \mathrm{UI} / \mathrm{kg} / 24 \mathrm{~h}$ \\
\hline
\end{tabular}

F: Forte. * Hasta $100 \mathrm{~kg}$. Presentaciones cuya dosis recomendada se ajusta a cada intervalo de peso. 
ciones de las distintas HBPM utilizadas en España. De todas formas, es posible, que la utilización en un futuro más o menos próximo de los nuevos antitrombóticos, como el fondaparinux (7) y el ximelagatrán (8) cambien radicalmente el tratamiento de la enfermedad.

Debe tenerse en cuenta que en los pacientes tratados con heparina carecen de valor las determinaciones de antitrombina $\mathrm{y}$ en los tratados con anticoagulantes orales (AO) no pueden valorarse los resultados de las proteínas $\mathrm{C}$ y $\mathrm{S}$.

\section{CASOS EN LOS QUE SE DEBEN UTILIZAR LAS HEPARINAS NO FRACCIONADAS (HNF) EN LUGAR DE LAS HBPM EN EL TRATAMIENTO DE LA TVP}

En pacientes con inestabilidad hemodinámica y/o trombosis iliofemoral masiva: administrar heparina no fraccionada (HNF), con una dosis inicial de $5.000 \mathrm{UI}$ o $80 \mathrm{UI} / \mathrm{kg}$ en bolo intravenoso (IV), seguido de $32.000 \mathrm{UI} / 24 \mathrm{~h}$ o $18 \mathrm{UI} / \mathrm{kg} / \mathrm{hora}$ de 7-10 días. La administración de AO debe comenzar entre los días 1 y 3 después del inicio del tratamiento con HNF, simultaneando ambos tratamientos durante 3-5 días, y deteniendo la heparinoterapia cuando se hayan alcanzado niveles terapéuticos (INR entre 2 y 3 ) durante dos días consecutivos.

\section{COMPLICACIONES POR EL USO DE LAS DISTINTAS HEPARINAS}

\section{HNF: COMPLICACIONES HEMORRÁGICAS (9)}

La administración de heparina conlleva el riesgo de producir hemorragias, debido a su acción anticoagulante, es decir, a la potenciación de la acción inhibidora de la antitrombina sobre la trombina, factor $\mathrm{X}$ activado (Fxa) y otras serinproteasas de la coagulación, así como a su capacidad de alterar la función plaquetaria y de aumentar la permeabilidad capilar. Por todo ello, si utilizamos estas heparinas deberemos de tener en cuenta las siguientes premisas:

- Los niveles plasmáticos de la HNF no son fácilmente predecibles debido a sus interacciones con las células endoteliales, macrófagos, plaquetas y proteínas plasmáticas, lo que da lugar a la necesidad de realizar controles analíticos durante su administración. Para ello se utilizan métodos indirectos como el tiempo de tromboplastina parcial activado (TTPA) o el tiempo de trombina. Estudios en animales y en humanos han demostrado que los niveles idóneos de heparina en plasma son aquellos que están en un rango de 0,3-0,7 UI/ml. Estos niveles se corresponden con un TTPA entre 1,5 y 2,5 veces el TPTA control. Dada la diferente sensibilidad a la heparina de las diversas cefalinas comerciales, se recomienda que cada laboratorio establezca el rango de valores de TTPA correspondiente a las concentraciones de heparina en plasma citados.

-En los enfermos con TVP que presentan anticuerpos antifosfolípidos, éstos se asocian a la presencia de anticoagulante circulante que alarga el TTPA, lo que dificulta la adecuación de las dosis de HNF dentro de rangos terapéuticos, por lo que sería preferible monitorizar midiendo los niveles de anti-Xa, ya que el anticoagulante circulante no interfiere con la determinación por técnicas amidolíticas de dicho inhibidor.

- Si la TVP aparece en el postoperatorio de una cirugía mayor, el tratamiento con HNF no debe comenzarse hasta transcurridas 12-24 h, e incluso debe retrasarse más si hay hemorragia en la zona quirúrgica. En estos casos debe prescindirse del bolus inicial, la dosis debe ser algo más baja que la habitual y los pacientes deben ser sometidos a vigilancia clínica y monitorización de laboratorio más estricta. Incluso, si hay riesgo hemorrágico elevado, puede considerarse la posibilidad de sustituir inicialmente el tratamiento anticoagulante por la colocación de un filtro temporal en la vena cava inferior.

Ante una complicación hemorrágica, la actitud terapéutica dependerá de su importancia. En el supuesto de una hemorragia menor y en puntos accesibles y no peligrosos, bastará suspenderla temporalmente, reiniciándola cuando haya desaparecido el sangrado. Por el contrario, las complicaciones hemorrágicas graves, tanto por su cuantía como por su localización requieren, además de la supresión del fármaco, una inmediata neutralización de la actividad de la heparina circulante, lo que se consigue mediante la administración de protamina Esta es una proteína catiónica que se une fuertemente a la heparina, la cual es aniónica, en una proporción de $1 \mathrm{mg}$ de protamina por cada 100 UI de HNF. Dado que la vida media de esta es algo superior a 1 hora cuando se administra a dosis terapéuticas, para una eliminación prácticamente total de la heparina circulante deberemos administrar una dosis de protamina correspondiente a la HNF perfundida en las últimas 2 horas. Posteriormente comprobaremos que la neutralización ha sido correcta a través de la normalización del TTPA. En el caso de administración de heparina por vía subcutánea, se requerirán dosis menores pero repetidas de protamina para neutralizar la heparina que progresivamente entra en la circulación general.

La administración de protamina IV debe hacerse a razón de 1-3 mg/m. para evitar la aparición de hipotensión o taquicardia.

\section{HBPM: COMPLICACIONES HEMORRÁGICAS (10)}

—La longitud de las cadenas de la heparina no influye en su efecto potenciador de la acción inhibidora de la antitrombina sobre el FXa, pero no ocurre así con la potenciación de su capacidad inhibidora de la trombina, para lo que se requieren cadenas con al menos 18 sacáridos. Debido a ello, las HBPM que tienen un gran contenido en cadenas de menos de 18 sacáridos, poseen una capacidad para potenciar la función anti-Xa de la antitrombina superior a la de potenciación de la inhibición de la trombina. En consecuencia, siempre que se emplee una $H B P M$, su dosis debe ajustarse utilizando la cuantificación de los niveles de anti-Xa, ya que carece de utilidad el uso de TTPA. Estos ajustes pueden ser necesarios sobretodo en pacientes con insuficiencia renal o con obesidad marcada.

-En caso de complicaciones hemorrágicas importantes también se hace necesaria la neutralización de su actividad mediante la administración de protamina. Esta, que neutraliza totalmente tanto la actividad antitrombina como la anti-Xa de la HNF, en el caso de las HBPM, neutraliza totalmente la actividad antitrombina pero sólo parcialmente la actividad antiXa debido a la menor afinidad de las HBPM por la protamina.

Aunque a nivel experimental la protamina detiene la hemorragia, carecemos de estudios en humanos que lo confirmen. A nivel práctico se recomienda que cuando la hemorragia se produce dentro de las primeras 8 horas tras la inyección 
de HBPM, se administre $1 \mathrm{mg}$. de protamina por cada $100 \mathrm{UI}$ anti-Xa en la última dosis administrada, y si persiste el sangrado se inyectará una segunda dosis de $0,5 \mathrm{mg}$ por cada 100 UI anti-Xa.

Se puede también transfundir plasma fresco: 3 unidades (15-20 ml/kg peso). Debe efectuarse un control de coagulación a los 60 minutos.

\section{COMPLICACIONES TROMBÓTICAS PROVOCADAS POR LA HEPARINA} (11)

\section{Trombocitopenia inducida por heparina (TIH)}

$1^{\circ}$ Tipo I: cursa con una moderada reducción del recuento plaquetario que, en general, se mantiene por encima de $100 \mathrm{x}$ $10 \% / 1$ y sin manifestaciones clínicas de ningún tipo. Suele presentarse entre el 2-4 día de tratamiento con heparina y se ha descrito hasta en un $30 \%$ de los pacientes tratados. Se cree que está producida por la acción directa de la heparina o sus contaminantes sobre las plaquetas y no está mediada por un mecanismo inmunológico.

$2^{\circ}$ Tipo II: suele presentarse tardíamente, entre 5 y 14 días después de iniciada su administración. Es de carácter inmune, secundaria a la producción de anticuerpos, generalmente IgG dirigidos contra el complejo heparina-factor plaquetario 4 (FP4).

Puede cursar con una trombopenia intensa: < 50 × 10\%/l, que en un $70 \%$ de los casos se asocia a trombosis arteriales, que pueden conducir a la amputación de un miembro, o a ETEV, con una mortalidad no despreciable. Por tanto es aconsejable controlar periódicamente la cifra de plaquetas durante las 2 primeras semanas de administración de heparina.

\section{Necrosis cutánea inducida por heparina}

Se presenta como placas rojizas muy dolorosas o como una necrosis franca cutánea en los puntos de inyección de la heparina, una 24 horas después de administrarla. Aparece al $5^{\circ}$ día de iniciada la administración de heparina y están asociadas a la presencia de anticuerpos dirigidos contra heparinaFP4. Sólo la cuarta parte de los pacientes con necrosis cutánea desarrollan trombopenia, pero cuando ésta aparece el cuadro suele presentar relevancia clínica por los fenómenos trombóticos arteriales en las extremidades.

\section{CONFIRMACIÓN DIAGNÓSTICA DE LA TIH TIPO II (12)}

Su confirmación diagnóstica se puede obtener mediante tres tipos de pruebas:

1. Demostración de anticuerpos contra heparina-FP4 por métodos inmunoenzimáticos, con una sensibilidad del 8090\%. Actualmente es la prueba de elección.

2. Activación de plaquetas normales por los anticuerpos del suero del paciente, en presencia de concentraciones de heparina entre 0,1 y $0,3 \mathrm{UI} / \mathrm{ml}$, valorando dicha activación mediante la liberación de serotonina plaquetaria marcada con 14C.

3. La prueba más simple: inducción de la agregación plaquetaria del sujeto sano en presencia del suero del paciente y de heparina. Lamentablemente la prueba es poco sensible, detectando en promedio sólo un $50 \%$ de los casos.

\section{TRATAMIENTO DE LA TIH TIPO II}

Administración IV de hirudina recombinante (lepirudina): bolo inicial de $0,4 \mathrm{mg} / \mathrm{kg}$. seguido de una perfusión de 0,15 $\mathrm{mg} / \mathrm{kg} /$ hora. No presenta reacción cruzada alguna con las heparinas. Control: TTPA Paciente/control, 1,5-2,5. Conjuntamente se administran AO, suspendiendo aquella cuando el INR esté entre 2-3.

Se puede utilizar el heparinoide danaparoid: bolo inicial IV de $2.500 \mathrm{U}$, seguida de una perfusión de 200-400 U/hora. No presenta reacción cruzada con la heparina en el $90 \%$ de los casos. Posee una vida media muy larga (25 horas), lo que debe tenerse en cuenta si se han de programar procedimientos cruentos. Su presencia en sangre no prolonga el tiempo de protrombina.

\section{TRATAMIENTO TROMBOLITICO DE LA TVP (13)}

La mortalidad secundaria a la TVP, una vez iniciada la heparinoterapia es muy baja, por lo que intentar reducirla no justifica la trombolisis, habida cuenta del riesgo hemorrágico que su administración conlleva. El objetivo principal de este tratamiento es la prevención del síndrome postrombótico (SPT), provocado por la incompetencia valvular secundaria a la trombosis. Se supone que una eliminación precoz del trombo que bloquea el aparato valvular venoso daría lugar a su recuperación funcional, lo que podría deducirse de algunos estudios angiográficos, pero que no está demostrado a nivel clínico. Aunque esta complicación puede llegar a ser invalidante para el paciente, no representa por sí misma, un riesgo de muerte, mientras que el propio tratamiento sí lo es.

Con la administración de trombolíticos, la lisis del trombo es 3,5 veces superior a la obtenida con heparina, aunque no está demostrado que por ello se prevenga el desarrollo posterior del SPT. Tampoco hay datos que confirmen que los trombolíticos reduzcan el riesgo de embolismos pulmonares (EP), pero si los hay de que su administración se asocia con un 1$3 \%$ de hemorragias intracraneales.

En resumen, la indicación de trombolisis en la TVP de los miembros inferiores es poco aceptada actualmente debido a que la relación riesgo-beneficio no es satisfactoria.

Una excepción, aceptada generalmente, serían las trombosis venosas masivas de miembros inferiores con riesgo de gangrena (flegmasia cerulea dolens), en pacientes con bajo riesgo hemorrágico. En este caso se pueden administrar cualquiera de los trombolíticos siguiendo las pautas recomendadas en la tabla III.

Previamente a su administración hay que efectuar un estudio básico de hemostasia, que incluya el recuento de plaquetas, para descartar aquellas alteraciones que puedan incrementar el riesgo hemorrágico provocado por el tratamiento, aunque si estas existen, probablemente, se reflejarán en una historia clínica bien realizada. Ésta es el principal elemento para descartar el uso de los trombolíticos, lo que se debe hacer teniendo en cuenta los apartados de la tabla IV.

Es sabido que existe poca correlación entre las pruebas de laboratorio para valorar la actividad fibrinolítica y el grado de 
TABLA III

INDICACIONES, FÁRMACOS Y POSOLOGÍA EN LA TERAPÉUTICA FIBRINOLÍTICA DEL TEV

\begin{tabular}{|c|c|c|c|}
\hline Indicación & Condiciones & Fármacos & Dosis \\
\hline \multirow[t]{3}{*}{ EP } & $\begin{array}{l}\text { Formas masivas (ocupación vascular } \\
>50 \% \text { ) con shock o inestabilidad } \\
\text { hemodinámica o sobrecarga ventricular } \\
\text { derecha grave }\end{array}$ & Alteplasa (rt-PA) & $\begin{array}{l}100 \mathrm{mg} \text { en } 2 \text { horas o } 0,6 \mathrm{mg} / \mathrm{kg} \\
\text { en } 15 \mathrm{~min} \text {. (máximo } 50 \mathrm{mg} \text { ) }\end{array}$ \\
\hline & & Urocinasa & $\begin{array}{l}4.400 \mathrm{U} / \mathrm{kg} / \mathrm{h} \text { x } 12 \mathrm{~h} \text { o } 15.000 \mathrm{U} / \mathrm{kg} \\
\text { en } 10 \mathrm{~min} \text {., en la aurícula derecha o } 3 \\
\text { millones de } \mathrm{Ul} \text { en } 2 \mathrm{~h}\end{array}$ \\
\hline & & Estreptocinasa & $\begin{array}{l}250.000 \mathrm{U} \text { en } 30 \mathrm{~min} \text { y } 100.000 \mathrm{U} / \mathrm{h} \mathrm{x} \\
24 \mathrm{~h}\end{array}$ \\
\hline \multirow[t]{3}{*}{ TVP } & $\begin{array}{l}\text { Trombosis masivas con riesgo de gangrena } \\
\text { (flegmasia cerulea dolens) }\end{array}$ & Estreptocinasa & $\begin{array}{l}250.000 \mathrm{U} \text { en } 10 \mathrm{~min} .100 .000 \mathrm{U} / \mathrm{h} \mathrm{x} \\
48-72 \mathrm{~h}\end{array}$ \\
\hline & & Urocinasa & $4.400 \mathrm{U} / \mathrm{kg} . / \mathrm{h} \times 24 \mathrm{a} 48 \mathrm{~h}$ \\
\hline & & Alteplasa (rt-PA) & $\begin{array}{l}0,5 \mathrm{mg} / \mathrm{kg} \text {, a pasar en } 4 \text { horas(Máximo } \\
150 \mathrm{mg} \text { ) }\end{array}$ \\
\hline
\end{tabular}

TVP: trombosis venosa profunda. EP: embolia pulmonar

\section{TABLA IV}

\section{CONTRAINDICACIONES DE LA TROMBOLISIS}

Contraindicaciones absolutas

Cualquier historia previa de AVC hemorrágico

Otro tipo de AVC durante el último año, excluyendo accidentes transitorios

Enfermedad intracraneal o intraespinal

Neoplasia cerebral o cirugía cerebral en los últimos 6 meses

Traumatismo craneal en las últimas 6 semanas (incluso leve si es en las últimas 48 horas)

Sospecha de disección aórtica

Pericarditis

Hemorragia interna en las últimas 6 semanas*

Sangrado activo (no menstruación) o diátesis hemorrágica conocida

Cirugía o trauma mayor en las últimas 6 semanas

\section{Contraindicaciones relativas}

Anticoagulación oral

Pancreatitis aguda

Embarazo o primera semana postparto

Úlcera péptica activa

Accidente isquémico transitorio en las últimas 6 semanas.

Demencia

Endocarditis infecciosa

Tuberculosis pulmonar activa cavitada

Enfermedad hepática avanzada

Evidencia de trombo intracardiaco

Hipertensión arterial (PA sistólica > 180 mm Hg o PA diastólica> $110 \mathrm{~mm} \mathrm{Hg}$ ) no controlable

Punción de un vaso no compresible en las 2 semanas previas

Maniobras traumáticas de resucitación en las últimas 3 semanas

*Valorar individualmente en caso de EP con alteración hemodinámica grave trombolisis obtenido o el riesgo clínico de hemorragias. Lo que es especialmente cierto en el caso del rt-PA, que no requiere control analítico alguno durante su administración. Al término de esta se instaurará la perfusión de heparina cuando la prolongación del TTPA del paciente sea menor del doble del valor control.

Con respecto a los fármacos con períodos de perfusión largos, como la urocinasa y la estreptocinasa, se puede realizar una determinación del TTPA dos a cuatro horas después de iniciar el tratamiento, cuya prolongación nos indicará que existe actividad fibrinolítica. La perfusión posterior de heparina seguirá los mismos pasos que la descrita para el rt-PA.

\section{MANEJO DE LAS COMPLICACIONES DE LA TERAPIA TROMBOLÍTICA}

- Si hay una hemorragia grave, se suspenderá su administración, incluida la heparina asociada. En este último caso, si la prolongación del TTPA > a 1,5 veces el TTPA control, habrá de neutralizarse la heparina circulante con protamina: 1 mg de protamina/100 UI heparina administradas en las dos últimas horas, aproximadamente.

— Si se está administrando un fibrinolítico de vida media larga (estreptocinasa), se neutralizará la actividad fibrinolítica mediante administración de ácido tranexámico (1 g IV), y no se procederá a la transfusión de sangre o hemoderivados hasta realizada ésta.

- Hay que tener en cuenta que la recuperación espontánea de la cifra de fibrinógeno es lenta, unas 24 h aproximadamente. Por ello, el riesgo hemorrágico puede persistir horas después de haber suspendido la administración de un activador de vida corta como el r-tPA. La administración de $3 \mathrm{~g}$. de fibrinógeno produce una elevación aproximada de $1 \mathrm{~g} / \mathrm{l}$ en el nivel de fibrinógeno plasmático.

Ocasionalmente puede ser necesario suspender el fibrinolítico (habitualmente estreptocinasa) por la aparición de reacciones alérgicas o febriles.

Cuando se utiliza un trombolítico de acción corta como el rt-PA, el TTPA se realizará inmediatamente después de su 
administración. Si se utilizan trombolíticos de acción larga (estreptocinasa, uroquinasa), el TTPA se determinará a las 2-4 horas después de iniciar el tratamiento.

\section{FILTROS DE VENA CAVA INFERIOR (VCI) EN EL TROMBOEMBOLISMO VENOSO (14)}

El tratamiento habitual de la enfermedad tromboembólica venosa aguda, es la heparinoterapia, seguida de los anticoagulantes orales. Sin embargo, este tratamiento puede estar contraindicado, ser insuficiente, o causar complicaciones que desaconsejen su utilización. En estos pacientes puede estar indicada la interrupción de la VCI para dificultar el paso de émbolos desprendidos de una TVP a la circulación pulmonar y así prevenir la muerte por EP.

Este tipo de filtros no están exentos de complicaciones, aunque existen situaciones bien definidas en las que está indicada su utilización:

1. En pacientes con contraindicaciones para la anticoagulación, hemorragias mayores durante la misma, o EP recurrentes, a pesar de llevar una anticoagulación adecuada.

2. En los casos de embolismo de repetición crónico con hipertensión pulmonar, y en pacientes que van a ser sometidos a embolectomía o endarterectomía pulmonar.

3. En el resto de las indicaciones no existen estudios bien diseñados que avalen su utilización sistemática, debiéndose valorar cada caso de forma individualizada.

4. Aunque sólo existen datos preliminares, la inserción de filtros temporales pueden ser de gran utilidad, sobre todo en aquellas situaciones en las que se prevea que la contraindicación para la anticoagulación será transitoria.

A pesar de no existir unas líneas claras y bien definidas sobre la necesidad ni el momento de asociar tratamiento anticoagulante en pacientes con filtro de VCI, así como tampoco de la duración del mismo una vez instaurado, parece razonable administrarlo tan pronto como sea posible para tratar el tromboembolismo venoso y disminuir la retrombosis.

\section{TRATAMIENTO QUIRÚRGICO DE LA TVP (15)}

Las heparinas son la estrategia terapéutica de elección para los pacientes con TVP. Sin embargo, en los casos que cursan con isquemia venosa, que generalmente, aparecen en las oclusiones más proximales -venas ilíaca y cava inferior-, no han demostrado su efectividad en todas las ocasiones. En estas situaciones el único tratamiento eficaz es restablecer rápidamente la permeabilidad venosa del eje del miembro, lo que difícilmente se puede conseguir por otro procedimiento que no sea la trombectomía venosa.

\section{La trombectomía venosa, está indicada en casos agudos de:}

TVP de los miembros inferiores que cursan con isquemia venosa del miembro o compromisos graves de retorno (flegmasia cerulea dolens) y algunas flegmasias alba dolens.

TVP de los miembros inferiores por obstrucción iliofemoral y/o cava en pacientes jóvenes

En los casos de TVP en embarazadas, a pesar de tratarse de enfermas jóvenes, no se realizará trombectomía salvo cuando exista una isquemia venosa.

La realización de fístula arteriovenosa adicional al terminar la trombectomía se practicará:

En los pacientes jóvenes en los que se realice trombectomía venosa y que padezcan una trombofilia, no ligando la fístula en estos casos, salvo que aparezcan signos y síntomas de alteraciones hemodinámicas generales o de sobrecarga local.

En cualquier indicación de trombectomía venosa sobre pacientes en los que exista contraindicación formal de anticoagulación y especialmente en los jóvenes.

En pacientes jóvenes, en los que esté indicada la trombectomía venosa pero no tengan trombofilia, la fístula arteriovenosa se instaurará, pero podrá ligarse al cabo de 3-12 meses, según la tolerancia clínica.

Para unos autores la fase aguda de la enfermedad no debe superar los 8 días, para otros incluso no más de 3. De cualquier forma, debe realizarse una evaluación ecográfica previa del trombo que siempre será más exacta que la valoración clínica de su antigüedad. En el supuesto de ser una trombosis crónica, será prácticamente imposible conseguir su evacuación completa, lo que puede abocar a una retrombosis en un elevado porcentaje de casos. Algunas veces no se consigue repermeabilizar el trayecto venoso ocluido. En estas situaciones se puede realizar un puente cruzado con fístula arteriovenosa realizada distalmente al origen de la arteria femoral superficial.

\section{Bibliografía}

1. Wheeler HB, Anderson FA. Diagnostic methods for deep vein thrombosis. Haemostasis 1995; 25: 6-26.

2. Wells P, Anderson D, Rodger M, Forgie M, Kearon C, Dreyer J, et al. Evaluation of D-Dimer in the Diagnosis of suspected Deep-Vein Thrombosis. N Engl J Med 2003; 349: 1227-1235.

3. Larsen TB, Stoffersen E, Christensen CS, Laursen B. Validity of Ddimer tests in the diagnosis of deep vein thrombosis: a prospective comparative study of three quantitative assays. J Intern Med 2002; 252: 3640.

4. Fraser JD, Andersosn DR. Deep venous thrombosis: Recent advances and optimal investigations with US. Radiology 1999; 211: 9-24.

5. Kakkar V, Gebska M, Kadziola Z, Roach C, Saba N, Manning A. Objective assessment of acute and long term treatment with unfractio-

nated heparin and low molecular weight heparin in acute deep vein thrombosis. Thromb Haemost 2001; 86 (Supl.): oc 967 (abstract).

6. Merli G, Spiro T, Olson C, Abildgaard V, Davidson B, Eldor A, et al, for the Enoxaparin Clinical Trial Gruop. Subcutaneous enoxaparin once or twice daily compared with intravenous unfractionated heparin for treatment of venous thromboembolic disease. Ann Intern Med 2001; 134: 191-202.

7. The Matisse Investigators. Subcutaneous fondaparinux versus intravenous unfractionated heparin in the initial treatment of pulmonary embolism. N Engl J Med 2003; 349: 1695-1702.

8. Schulman S, Wahlander K, Lundström T, Clason S, Eriksson H. Secondary prevention of venous thromboembolism with the oral direct thrombin inhibitor ximelagatran. N Engl J Med 2003; 349: 1713-1721. 
9. Hirsh, Warkentin T, Shaughnessy S, Annand S, Halperin JL, Raschke $\mathrm{R}$, et al. Heparin and low-molecular-weight heparin: mechanisms of action, pharmacokinetics, dosing, monitoring, efficacy, and safety. Chest 2001; 119 (1 Suppl): 64S-94S.

10. Dolovich LR, Ginsberg JS, Douketis JD, Holbrook AM, Cheah G. Meta-analysis comparing low-molecular-weight heparins with unfractionated heparin in the treatment of venous thromboembolism: examing some unanswered questions regarding location of treatment, product type, and dosing frequency. Arch Intern Med 2000; 160: 181-188.

11. Amiral J, Bridey F, Wolf M, Boyer C, Fressinaud E, Vissac AM. Antibodies to macromolecular platelet factor 4-heparin complexes in heparin-induced thrombocytopenia: a study of 44 cases. Thromb Haemost 1995; 73: 21-28.

12. Greinacher A, Amiral J, Dummel V, Vissac A, Kiefel V, Mueller Eck- hardt C. Laboratory diagnosis of heparin-associated thrombocytopenia and comparison of platelet aggregation test, heparin-induced platelet activatión test, and platelet factor 4 / heparin enzyme-linked immunosorbent assay. Transfusion 1994; 34: 381-385.

13. Ginsberg JS, Hirsh J. Treatment of venous thromboembolism. In: Colman RW, Hirsh J, Marder J, Clowes AW, Georg JN, editors. Haemostasis and Thrombosis. Philadelphia: Lippincot Williams \& Wilkins, 2001, p. $1405-1412$

14. Caprini J, Arcelus J, Ryna J. Effective risk stratification of surgical and non surgical patients for venous thromboembolic disease. Semin Hematol 2001; 38 (Supl. 5): 12-19.

15. Plate G, Eklof B, Norgren L, Ohlin P, Dahlstrom JA. Venous thrombectomy for iliofemoral vein thrombosis-10-year-results of a prospective randomised study. Eur J Vasc Surg 1997; 14: 367-374. 